University of Wollongong

Research Online

Faculty of Informatics - Papers (Archive)

Faculty of Engineering and Information

Sciences

June 2006

\title{
An Overview on the Integrated IP Optical Data Control Plane in the Optical Transport Network
}

S. Chen

University of Wollongong

Follow this and additional works at: https://ro.uow.edu.au/infopapers

Part of the Physical Sciences and Mathematics Commons

\section{Recommended Citation}

Chen, S.: An Overview on the Integrated IP Optical Data Control Plane in the Optical Transport Network 2006.

https://ro.uow.edu.au/infopapers/474

Research Online is the open access institutional repository for the University of Wollongong. For further information contact the UOW Library: research-pubs@uow.edu.au 


\title{
An Overview on the Integrated IP Optical Data Control Plane in the Optical Transport Network
}

\author{
Abstract \\ In recent years, the Optical Transport Network (OTN) systems have been introduced widely, however, wide \\ adoption has been limited due to the immature infrastructural issues. The OTN infrastructure takes the \\ responsibility of data and signal transmission for substratal layer, as well as provides carrying and \\ maintaining services (mostly the IP-based traffic today) through an optical data transport control plane. \\ Thus, this paper focuses on the latest OTN data control plane and therefore gives a comprehensive \\ introduction on the integrated IP Optical peer control plane.

\section{Disciplines} \\ Physical Sciences and Mathematics

\section{Publication Details} \\ This paper was originally published as: Chen, S, An Overview on the Integrated IP Optical Data Control \\ Plane in the Optical Transport Network, Proceedings of the 2006 International Conference on \\ Communications, Circuits and Systems, Guilin, Guangzi, China, June 2006, 3, 1872-1876. Copyright 2006 \\ IEEE.
}




\title{
An Overview on the Integrated IP Optical Data Control Plane in the Optical Transport Network
}

\author{
Sheng Chen \\ School of Information Technology and Computer Science \\ University of Wollongong \\ Wollongong, NSW 2522, Australia \\ sc960@uow.edu.au
}

\begin{abstract}
In recent years, the Optical Transport Network (OTN) systems have been introduced widely, however, wide adoption has been limited due to the immature infrastructural issues. The OTN infrastructure takes the responsibility of data and signal transmission for substratal layer, as well as provides carrying and maintaining services (mostly the IP-based traffic today) through an optical data transport control plane. Thus, this paper focuses on the latest OTN data control plane and therefore gives a comprehensive introduction on the integrated IP Optical peer control plane.
\end{abstract}

\section{INTRODUCTION}

The data control plane decides the capability and reliability of the OTN infrastructure. Based on existing IPoriented multiservice architecture, the first phase for deployment of the OTN is to set up a static optical layer under the IP layer. Because the light paths utilized in this model are assigned manually and statically, this structure is also called Static IP Optical Overlay Control Plane. All data traffic are converged at the IP network edge and converted into optical signals, and simply transferred along the preconfigured optical route.

However, with the increasing commercial deployment of advanced optical transport components such as Optical Add/Drop Multiplexers (OADMs) and Optical CrossConnects (OXCs), the upper layer IP traffic in most current OTNs are converged at the WDM (Wavelength Division Multiplexing)/DWDM (Dense WDM) junction points, and dynamically switched into multiple wavelengths with different types of service classified. This is also referred as optical routing or wavelength routing. Network services in same categories can be routed and switched on the physical wavelength domain during the transportation, rather than by back to the IP environments. Hence, this data traffic control method, which is based on Dynamic IP Optical Overlay Control Plane, enhances the OTN with further efficiency and survivability.

Because of the complex IP NGN (Next Generation Network) service architecture, including the integration of data, voice and video from future network requirements, large service providers and corporate network operators are seeking for a more flexible and dynamic solution on setting up connections, assigning bandwidth and managing wavelength resources of the OTN system. Therefore, future trends of the OTN control mechanism will centre on the convergence of the dynamic OTN wavelengths (services) distribution and the intelligent IP traffic routing. Based on this principle, the optical layer are expected to communicate with the IP networks at the same control plane directly, namely the Integrated IP Optical Peer Control Plane, which offers the network control complete transparency between the two layers.

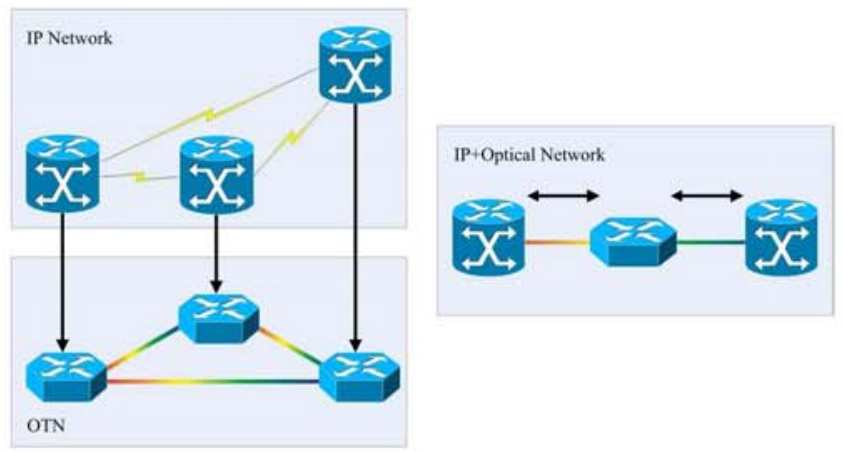

Figure 1. IP/Optical overlay model and IP/Optical peer model

When comparing the three types of control plane (see Figure 1), it is easy to find that the static IP overlay and the dynamic IP overlay optical control plane are similar in several aspects. For instance, the optical layer in both models is opaque to the IP layer, which means the OTN just provides basic connection basement for upper layer IP networks. In particular, the lightpath selecting and assigning mechanisms in these overlay structures is not or part under the control of the IP layer [1]. By contrast, the peer model aims on providing a more efficient and flexible control method, to establish an intervenient uniform control platform between the optical and the IP layer. In this ideal approach, 
the optical wavelength and bandwidth allocation can be managed by IP routing transparently.

\section{Basic Principle Of WaVElength Routing}

With the increasing and changeful demands of business operations, the static assigned optical domain faces the problem of a considerable amount of manual maintenance tasks such as adding new wavelength channels with new services or reconfiguring the light paths when network failures. Thus, a dynamic wavelength routing approach combined with the classical IP routing concept (see Figure 2) is required for fulfilling the future requirements of intelligent optical internetwork. In doing so, the wavelength channel selecting, connecting and dropping mechanisms are accomplished by the $\mathrm{OXC}$ and the wavelength routing controller, or the combination of both modules, namely the wavelength router (WR).

Generally, classical IP routing protocols such as OSPF and IS-IS are applied to generate a wavelength routing protocol, which assists the WRs to dynamically connect IP routers or other upper layer service equipments. The WRs take charge of maintaining a cross-connect table consists of information about input and output fibre port, and input and output channel of DWDM modules, which is similar as the functions of IP routing table inside IP routers (see Figure 3). Furthermore, each of the active optical interfaces must have a valid IP address with appropriate subnet information for the WRs to communicate with optical layer neighbors and upon layer IP devices. As described in RFC3717, the IP routers and wavelength routers interact as in the client/server architecture and this also can be referred as the overlay model [2].

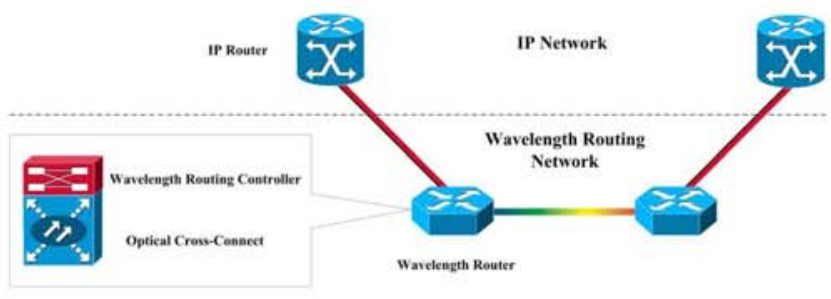

Figure 2. Wavelength routing network

When an optical channel trail (OCH trail) consists of an original wavelength throughout, the whole connection is called a wavelength path (WLP) [3]. In most cases, however, the data traffic is transported across a unidirectional lightpath, which contains different converted wavelengths from every individual network segments. In managing the provision of those service lightpaths, there are two common methods. The first method relies on a centralized management server maintains a database, including all the wavelength routing elements such as topology, network devices and other resources. Although this gives a clear view of the connection status and bandwidth allocation, it has a lack of management scalability and reliability. Thus, the second method aims on providing a distributed wavelength routing mechanism for those large scale OTN projects. Similar as the distance vector IP routing approach, each WR keeps a local wavelength routing table and exchanges the database with their neighbors, which allows all the routers to see the entire topology and to calculate a best lightpath independently and locally. In addition, with the altitudinal flexibility of wavelength allocation, the dynamic wavelength routing model is capable to provide bandwidth control mechanisms such as traffic classification and congestion management by assigning different service level agreements (SLAs) into specific wavelengths.

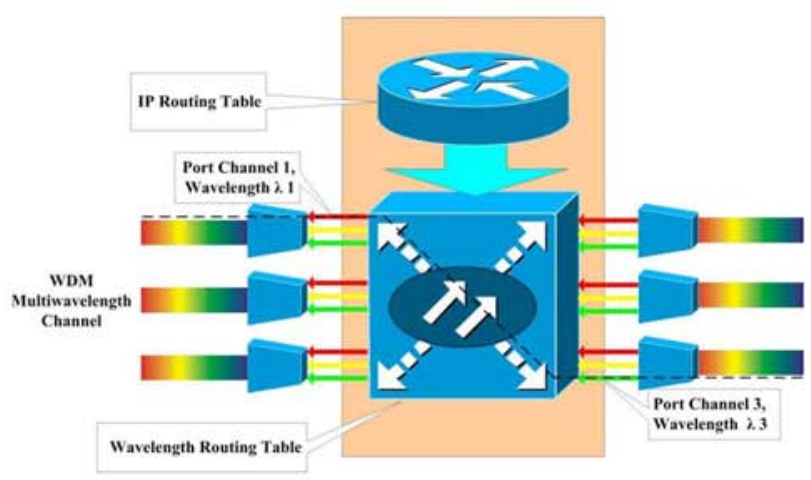

Figure 3. Basic operations of wavelength routing mechanism

\section{The Integrated IP Optical PeEr Control Plane}

\section{A. The integrated IP/MPLS peer control model}

Both the static and the dynamic overlay control models have a common point that the network components from the IP layer can not communicate with that from the OTN layer directly, in terms of the routing and wavelength assignment (RWA). However, there is little doubt that the future orientation of OTN development process is to achieve the ideal integrated peer model, which means the IP network and the OTN share and exchange control information at an identical plane. The concept of 'peer model' can be traced back to the popular age of the ATM system [4]. As ATM first provided the powerful cell switching capacity with outstanding QoS mechanisms, it has been deployed in backbone networks as a foundational transport technology rapidly. With the rising demands of various IP-oriented data services, large companies and ISPs then have to move their IP networks onto the ATM infrastructure. In connecting the two different networks, IP packets are routed and terminated at the intervenient network edges, and then encapsulated into cell formats for entering into the ATM switching domain. It is important to note that the IP routers have a lack of understanding about the particular ATM path assignment information such as the detailed PVC and SVC structures. Thus, an advanced transport control technology named Multiprotocol Label Switching (MPLS) is produced for fulfilling the gap and to enable a complete transparent communication between IP and ATM as peer neighbours. 


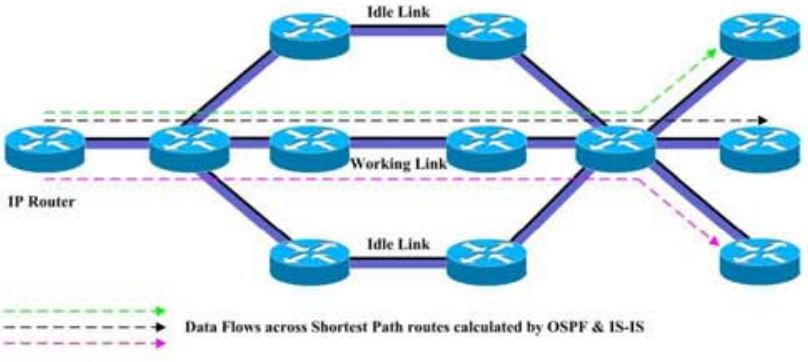

Figure 4. Standard IP routing causes wastage of network link resources

MPLS is a multilayer transport technology that functions between network layer and data-link layer, ordinarily in an IP-based core network. Generally, MPLS utilizes mature routing protocols such as OSPF or IS-IS, to generate an optimized IP routing table at the backbone edge. In the mean while, it operates a simple and efficient label switching mechanism inside the entire core network, instead of complex routing-based data forwarding. Under this approach, routers run MPLS are called label-switched routers (LSRs), which set up several label-switched paths (LSP) around interior backbone network. The data packet carries a label as a substitute of IP-header for implementing path oriented switching. As a rule, classical IP-based routing algorithms only select a lowest metric path as the best route (see Figure 4). This usually wastes of other available link resources and bandwidth. Conversely, MPLS not only performs an excellent traffic loading balance, but also intelligently prevents a link from becoming congested whereas other routes are idle (see Figure 5).

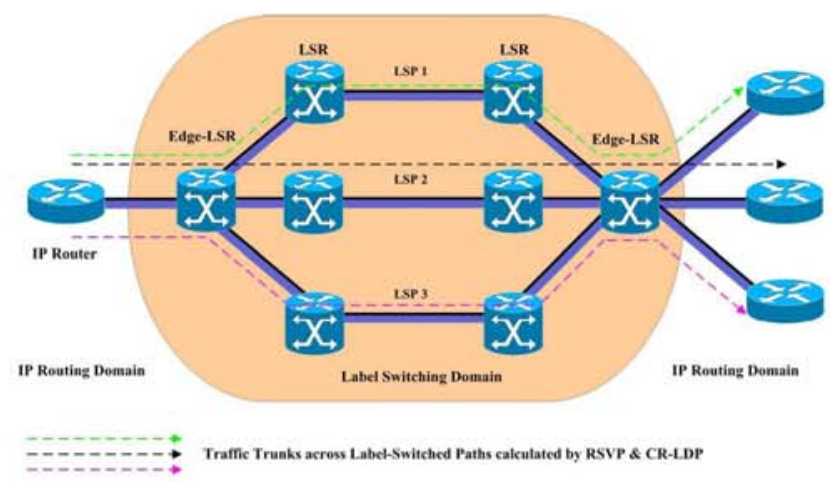

Figure 5. MPLS optimizes network utilization with specific QoS guarantees

\section{B. Advanced topics of QoS in the OTN}

As discussed above, MPLS introduces an innovative bandwidth control mechanism in IP-oriented data network. However, existing telecommunication core infrastructures involve a very complex network environment, including IP routers, routers with TDM (ATM/FR) interfaces, OADMs and OXCs. Under this circumstance, how to optimally utilize existing network structures and resources to achieve classbased traffic optimization is becoming the limelight of the advanced network QoS topics.

Previously, the network operators are used to put different metrics on specific IP network interfaces or ATM VCs as the basic Traffic Engineering (TE) implementations. However, the feature of combing MPLS with TE (MPLS-TE) improves every aspect of TE functionalities including optimizing network utilization, handling unexpected congestion as well as link and node failure. For setting up the LSPs in MPLSTE network at first, label distribution protocols (LDPs) such as RSVP and CR-LDP are applied to seek and establish endto-end guaranteed bandwidth tunnels for various high performance services [5]. Those services involve real-time multimedia applications such as audio/video conferencing, online multicasting, as well as data traffic from network file system (NFS) and VPN and so on.

For ensuring strategy level QoS planning in terms of various types of data services, the differentiated service (Diffserv) architecture is recognized as an ideal solution. Differserv architecture empowers traffic conditioning such as data flow marking, policing, shaping mechanisms, and the execution of per-hop behaviours (PHBs) such as traffic queuing, scheduling and dropping approaches [6]. In a MPLS-TE core network environment, every hop holds and shares the information with their neighbours about a set of policy labels, which differentiate all data packets with particular QoS behaviours. After receiving the packets from upstream nodes, local LSRs pop out the labels and implement various queuing and dropping activities as defined in the PHBs, by utilizing integrated specialized software. Besides, the LSRs also push their own policy settings (PHBs) into the label stacks and send them with the packets to the downstream neighbours (see Figure 6). Hence, a comprehensive TE approach ensuring global QoS strategy with sufficient efficiency and fairness is achieved.

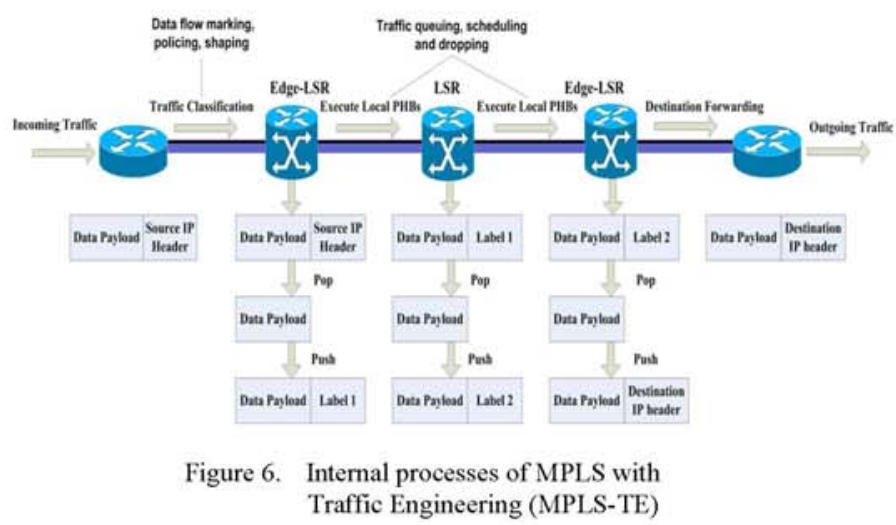

According to this traffic optimization principle, it is reasonably to extend the MPLS-TE into the optical domain. 
Thus, there was an innovative concept named Mutiprotocol Lambda Switching (MPLmS) extending label switching functionality from electrical to wavelength control plane. MPLmS was proposed to assign a wavelength to optical channel in the WDM-based core network, similarly as MPLS assigns a label to a LSP in the circuit-based IP backbone. This approach not only enhanced network resource control and management in the OTN, but also expanded the TE implementation from IP to optical domain. For instance, a practical deployment method is to label different clusters of Diffserv flows with different wavelengths at the edge between IP network and OTN (see Figure 7). Thus, the internal TE characteristics can be seamless inherited.

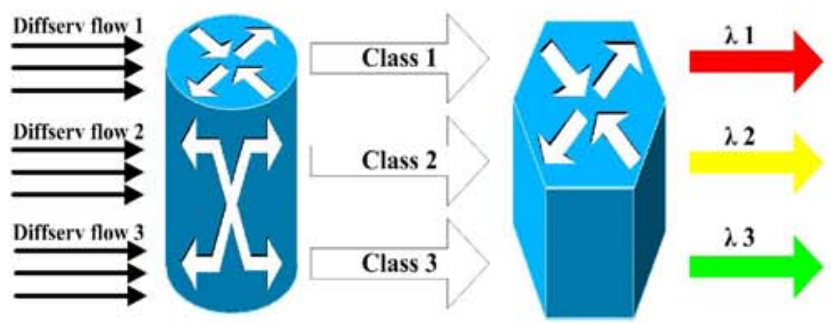

Figure 7. TE implementation from IP to Optical domain

\section{The intergration of the IP/MPLS and the OTN}

As MPLS was so successful on IP-based network QoS administration in terms of TE implementation, IETF has then generated the Generalized Multiprotocol label Switching (GMPLS) as an actualization of the MPLmS. GMPLS is indeed an extension of MPLS from IP to optical world, and it first enables the control and management of Packet Switch Capable (PSC) layer, Time Division Switch Capable (TDM) layer, Lambda Switch Capable (LSC) layer, as well as Fibre Switch Capable (FSC) layer within a common environment [7]. GMPLS supports all convergence forms of IP and optical: both the overlay model and the integrated peer model, which is flexible and scalable for deployment on different OTN environments.

The basic operation principle of GMPLS is very similar as that of MPLS, however, there is still some distinctions on the ways of provisioning the LSPs between them. For supporting the future demands of OTN, GMPLS introduces a novel label assignment and distribution architecture. For example (see Figure 8), the PSC layer labels are exchanged within all IP routers that stand at the outmost edge of the whole GMPLS domain; the TDM layer labels are exchanged between SONET/SDH and ATM equipments in the middle area; and the LSC/FSC layer labels are only exchanged between interfaces of OADMs and OXCs in the core. In brief, GMPLS LSP architecture is in a position to convert and allocate different types of services from external IP networks into different time slots in TDM infrastructure into different wavelengths/fibres at the core optical plane. As a consequence, this generalized label provision method provides a scalable TE solution with excellent management of diversified network resources including IP routes, time slots, wavelengths and fibres.

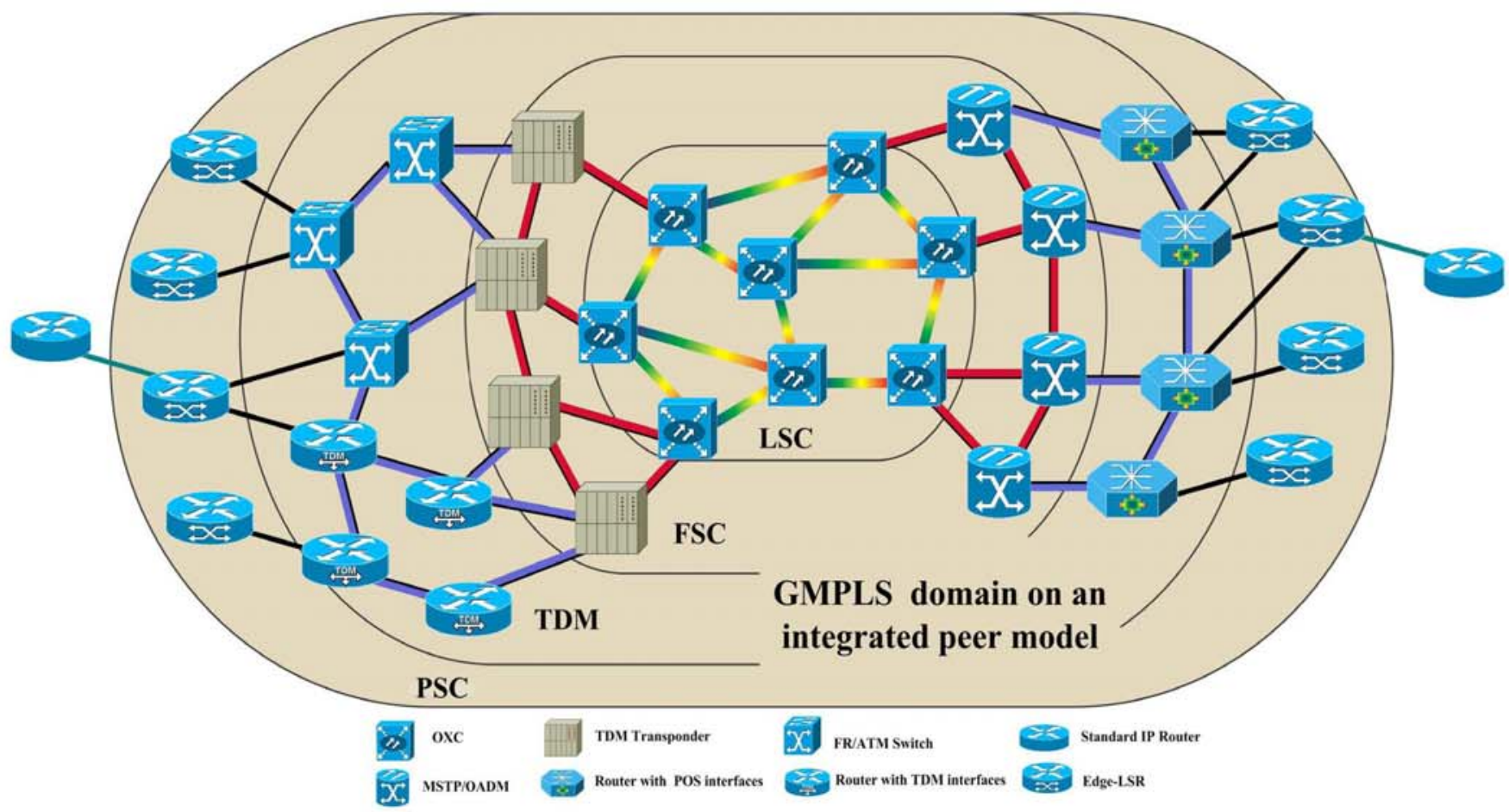

Figure 8. GMPLS domain on an integrated peer model 
In satisfying OTN-oriented considerations such as bidirectional communication of fibre links, GMPLS also indicates a bi-directional label distribution structure. Previously, the path selection in bi-directional OTN links is calculated separately, which increases the complexity and potential maintenance problems. Thus, there is a rapid increasing demand of consummating the optical LSP assignment mechanism, especially for those optical ISPs and optical intranet operators. In doing so, GMPLS utilizes a uniform signaling message structure for establishing bidirectional optical LSPs on both downstream and upstream, which reduces the setup latency and control overhead. This important feature, in addition, ensures both directional LSPs with symmetric TE requirements including fate sharing, protection and restoration, LSRs, and resource requirements [8]'.

Nevertheless, by utilizing extended TE-based resource control signaling such as Generalized RSVP and Generalized CR-LDP, GMPLS is able to establish end-to-end nonblocking bandwidth tunnels (LSPs) through from packet switching to TDM switching to Lambda/Fiber switching domains [9]. GMPLS focuses on the overall survivability of extended TE tunnels between head-end nodes, regardless of any intermediate network (IP, TDM or WDM) failure within an integrated peer control domain. By exchanging standard TE extension signaling, each network element in front of a failure will automatically bypass the protected services through local restoration link, path or tunnel. This feature also enables optical ISPs to provide services with highest guaranteed QoS level, as well as global ensured reliability through the entire network environment.

\section{CONCLUSION}

For more than twenty years research and development, IP has settled its absolute position in this industry as a leading internetwork standard. Current and future corporate intranets and ISP backbones are going to follow the trends to the efficient and cost-effective IP-based integrated multiservice architecture. Amid the ongoing evolution into optical networking, many standardization organizations are working jointly for enhancing the interoperability and maximizing the utilization of network resources between the OTN and the IP layer. Thus, it is reasonable to believe that the integrated IP optical peer model will largely unify the data control plane in future optical communication infrastructure.

\section{ACKNOWLEDGMENT}

I want to thank my supervisors, Ms. Gene and Dr. Katina for their precious support all the while. There is also a special thanks to Mr. Jian Ma, who is a system engineer in Cisco Systems, and other companions when I was in the project of SMEPC.

\section{REFERENCES}

[1] P. Tomsu, and C. Schmutzer, Next Generation Optical Networks: The convergence of IP Intelligence and Optical Technologies, Prentice Hall PTR, 2002.

[2] IETF. "IP over Optical Networks: A Framework", RFC3717, 2004.

[3] ITU-T. "Architecture of Optical Transport Network", G.872, 2003.

[4] IETF. "IP over ATM: A Framework Document", RFC1932, 1996.

[5] IETF. "Use of Interior Gateway Protocol (IGP) Metric as a second MPLS Traffic Engineering (TE) Metric", RFC3785, 2004.

[6] IETF. "An Architecture for Differentiated Services", RFC2475, 1998.

[7] K. Youngtak et al. "GLASS (GMPLS Lightwave Agile Switching Simulator) - A Scalable Discrete Event Network Simulator for GMPLS-based Optical Internet", Advanced Network Technologies Division, National Institute of Standards and Technology,USA. pp 2, 2002.

[8] IETF. "Generalized Multi-Protocol Label Switching (GMPLS) Signaling Functional Description", RFC3471, 2003.

[9] IETF. "RSVP-TE Extensions in support of End-to-End Generalized Multi-Protocol Label Switching (GMPLS)-based Recovery", Internet draft, April 2005. 